\title{
Biología y diversidad genética del SARS-CoV-2, agente causal de la COVID-19.
}

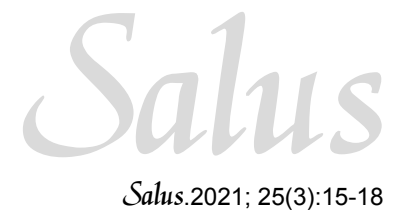

Biology and genetic diversity of SARS-CoV-2, the causative agent of COVID-19.

https://doi.org/10.54139/salus.v25i3.127

Rossana C Jaspe ${ }^{1}$ (D) José Luis Zambrano ${ }^{\text {(D) }}$ Carmen L Loureiro ${ }^{1}$ (D) Yoneira Sulbaran ${ }^{1}$ (D) Zoila C Moros ${ }^{2}$ (D) Domingo Garzaro $^{1}$ (D) Héctor R Rangel ${ }^{1}$ (D) Flor H Pujol $^{1}$ (D)

\section{RESUMEN}

La infección generada por el SARS-CoV-2 ha causado más de 200 millones de casos y de 4,5 millones de muertes en todo el mundo. EI SARS-CoV-2 ha ido acumulando mutaciones que permiten clasificarlo en diferentes linajes. Algunos de esos linajes han sido denominados variantes por la OMS: bajo vigilancia (VUM), de interés (VOI) o de preocupación (VOC). Metodología. Se han descrito diferentes estrategias para la vigilancia genómica de estas variantes del SARS-CoV-2 en cada país. En Venezuela las estrategias incluyen la amplificación de un fragmento de la espiga, PCR-RFLP y secuenciación del genoma completo viral, lo que nos ha permitido monitorear la introducción de VOCs y VOls al país. Resultados. Para octubre de 2021, en Venezuela se han descrito la circulación de tres VOCs, Alpha, Gamma y Delta, y las dos VOls (Lambda y Mu). Globalmente, la variante Delta predomina en prácticamente en todos los continentes salvo algunos países de Latinoamérica, aunque se estima que pronto prevalecerá también en la región. Discusión. La circulación de variantes en los países es un proceso muy dinámico y Venezuela no escapa de esta realidad; por ello es importante seguir la vigilancia genómica de este virus.

Palabras clave: Coronavirus, COVID-19, Variantes, Mutaciones, Vigilancia genómica

\section{ABSTRACT}

The infection generated by SARS-CoV-2 has caused more than 200 million cases and 4.5 million deaths worldwide. SARS-CoV-2 has accumulated mutations that allow it to be classified into different lineages. Some of these lineages have been designated variants by

1 Laboratorio de Virología Molecular, Centro de Microbiología y Biología Celular, Instituto Venezolano de Investigaciones Científicas, Caracas, Miranda, Venezuela.

${ }^{2}$ Laboratorio de Biología de Virus, Centro de Microbiología y Biología Celular, Instituto Venezolano de Investigaciones Científicas, Caracas, Miranda, Venezuela

Autor de correspondencia: Flor H Pujol (D)

E-mail: Ifhpujol@gmail.com

Recibido: 29-10-2021

Aprobado:27-11-2021 the WHO: under monitoring (VUM), of interest (VOI), or of concern (VOC). Methodology. Different strategies for genomic surveillance of these SARS-CoV-2 variants have been described in each country. In Venezuela, the strategies include the amplification of a fragment of the spike, PCR-RFLP, and sequencing of the complete viral genome, which has allowed us to monitor the introduction of VOCs and VOls to the country. Results. By October 2021, in Venezuela, the circulation of three VOCs, Alpha, Gamma, and Delta, and the two VOls (Lambda and Mu) have been described. Globally, the Delta variant predominates in practically all continents except some Latin American countries, although it is estimated that it will soon prevail in the region as well. Discussion. The circulation of variants in the countries is a very dynamic process and Venezuela does not escape from this reality; therefore, it is important to continue genomic surveillance of this virus.

Keywords: Coronavirus, COVID-19, Variants, Mutations, Genomic surveillance

\section{INTRODUCCIÓN}

En marzo de 2020 se declaró la pandemia de COVID-19 provocada por un coronavirus emergente, el SARS-CoV-2. A dos años de su identificación, esta nueva infección ha causado más de 200 millones de casos y de 4,5 millones de muertes en todo el mundo. Este artículo pretende describir la diversidad de este nuevo virus y la circulación de variantes en Venezuela.

Biología y diversidad del SARS-CoV-2. Este virus pertenece a la familia Coronaviridae, orden Nidovirales. Estos virus poseen una envoltura que rodea una nucleocápside helicoidal que contiene un gran genoma de ARN continuo de polaridad positiva de casi 30.000 nucleótidos (nt), que codifica 4 proteínas estructurales (nucleocápside o $\mathrm{N}$, espiga o $\mathrm{S}$, matriz $\circ \mathrm{M}$ y envoltura $\circ \mathrm{E}$ ), 15 proteínas no estructurales y 8 proteínas accesorios (Figura 1).

Este orden viral es único entre los virus de ARN en poseer una exonucleasa que habilita la capacidad de corrección de la replicación genómica, limitando así los eventos mutacionales. Sin embargo, el gran número de eventos de replicación que ha sufrido este virus, permitiendo la generación de genomas híbridos, una elevada frecuencia de recombinación, y la probable acción de las desaminasas del hospedero sobre el genoma viral (1). Esto ha permitido la aparición de muchas mutaciones puntuales y deleciones frecuentes en el genoma viral (2). 


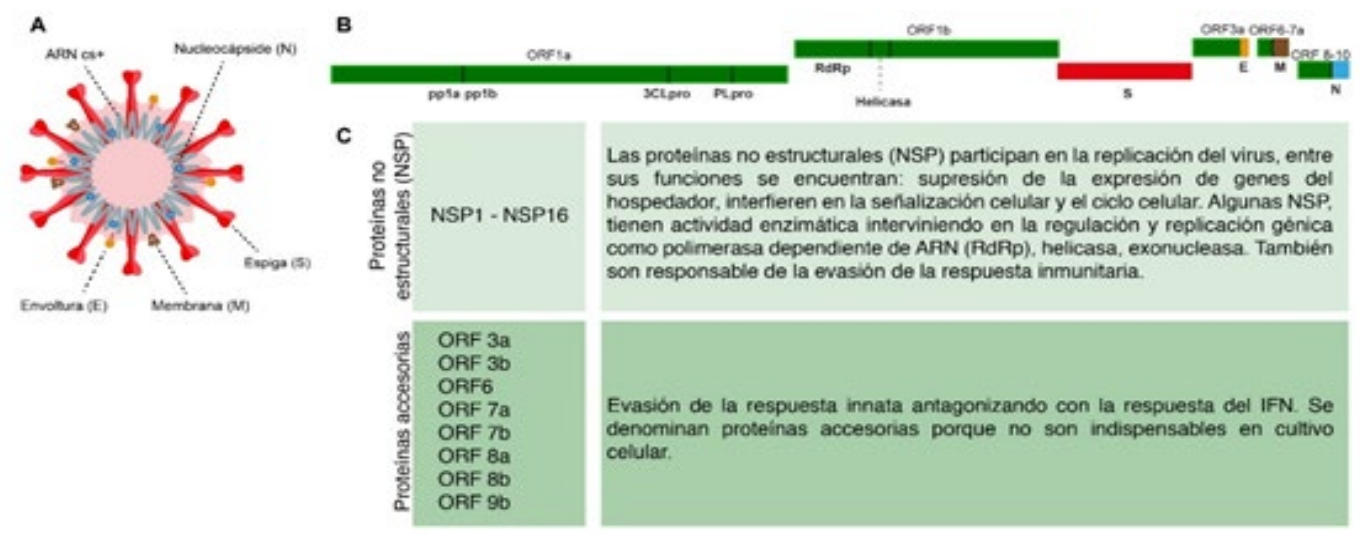

Figura 1. Organización genómica del SARS-CoV-2 y estructura del virión. A) Se señalan las 4 proteínas estructurales que conforman el virión: 100 copias de la proteína S, 20 de la proteína E, 2000 de la proteína M y 1000 de la proteína N, B) Organización del genoma ARN monocatenario de sentido positivo de $30.000 \mathrm{~Kb}$ y C) Proteínas no estructurales y accesorias y su función (1).

Variantes del SARS-CoV-2. El SARS-CoV-2 ha ido acumulando mutaciones que permiten clasificarlo en diferentes linajes y la clasificación más aceptada es la de Pangolin (3). Desde finales de 2020 han surgido también diferentes variantes, que son linajes de virus que comparten tipos particulares de mutaciones.

Algunas de estas variantes han sido definidas como bajo vigilancia (VUM), de interés (VOI) o de preocupación (VOC) por la OMS. La VUM es una variante con cambios genéticos que se cree pueden afectar las características del virus, puede evolucionar rápidamente, ser un riesgo a futuro pero sin evidencias fenotípicas o epidemiológicas de impacto, aunque requieren estar bajo vigilancia. Las VOI albergan mutaciones que pueden conferir a estos grupos de virus más transmisibilidad, o resistencia parcial a inmunidad protectora o tratamiento, entre otros.

Cuando se demuestran algunas de estas características para una VOI en particular, esta VOI se denomina VOC (4). Para octubre de 2021, las dos variantes designadas como VOI son la C.37 (Lambda, detectada por primera vez en Perú en Diciembre de 2020 y la variante del linaje B.1.621 (Mu), documentada por primera vez en Colombia en Enero 2021. La variante del linaje B1.1.7 (Alpha), para la que se observó un aumento de la incidencia después de su aparición en el Reino Unido, variante del linaje B.1.351 (Beta), con un aumento de la prevalencia en Sudáfrica, la variante del linaje B.1.1.28.1 o P.1 (Gamma) y la variante del linaje B.1.617.2 (Delta) son las 4 variantes de preocupación (Tabla 1). El primer nombre corresponde al linaje según la clasificación de los linajes Pango (4) y la letra griega corresponde a la clasificación de la OMS (5).

Algunas de las mutaciones presentes en estas variantes son motivo de preocupación para la salud pública por dos razones principales: la mutación N501Y (presente en las VOCs Alpha, Beta y Gamma), está asociada a un aumento de la transmisibilidad. Las mutaciones E484K y K417N/T (presente en las VOCs Beta y Gamma), se han asociado con frecuencia con casos de reinfección y podrían reducir la actividad neutralizante de los anticuerpos producidos por la vacunación. Las mutaciones L452R y P681R (presente en la VOC Delta) aumentan tanto la capacidad de transmisión como la evasión a la respuesta inmune protectora (5-8) (Tabla 1).

Tabla 1. Variantes de preocupación (VOCs) del SARS-CoV-2 (8)

\begin{tabular}{|c|c|c|c|c|}
\hline Variante & Alpha & Beta & Gamma & Delta \\
\hline Linaje & B.1.1.7 & B.1.351 & B.1.1.28.1 & B.1.617.2 \\
\hline País de emergencia & Reino Unido & Suráfrica & Brasil & India \\
\hline Fecha de designación OMS & $18-12-2020$ & $18-12-2020$ & $11-01-2021$ & $11-05-2021$ \\
\hline Mutaciones importantes & $\begin{array}{l}\text { E484K }{ }^{1} \\
\text { N501Y } \\
\text { D614G } \\
\text { P681H }\end{array}$ & $\begin{array}{l}\text { K417N } \\
\text { E484K } \\
\text { N501Y } \\
\text { D614G } \\
\text { A701V }\end{array}$ & $\begin{array}{l}\text { K417N } \\
\text { E484K } \\
\text { N501Y } \\
\text { D614G } \\
\text { H655Y }\end{array}$ & $\begin{array}{c}\text { L452R } \\
\text { T478K } \\
\text { D614G } \\
\text { P681H }\end{array}$ \\
\hline Transmisibilidad & Incrementada & Incrementada & Incrementada & $\begin{array}{c}\text { Incrementada } \\
\text { (la más alta descrita) }\end{array}$ \\
\hline Evasión RP² & No & Parcial & Parcial & Parcial \\
\hline Gravedad $^{3}$ & Posible incremento & No confirmado & No confirmado & Posible incremento \\
\hline
\end{tabular}

${ }^{1}$ La variante puede presentar o no está mutación. ${ }^{2} \mathrm{RP}$ : Respuesta inmunitaria protectora. ${ }^{3}$ Se sospecha un incremento en el riesgo de hospitalización para todas las variantes, aunque existe evidencia mayor para la Alpha y la Delta. 
Variantes del SARS-CoV-2 en Venezuela. Durante el año 2020 circularon varios linajes del SARS-CoV-2 en Venezuela. Desde el inicio de la epidemia en el país, la mutación D614G, una mutación que probablemente confirió mayor transmisibilidad al virus, se identificó de forma mayoritaria en los aislados circulantes (9). La mutación D614G también se encuentra en la proteína $S$ del virus, más no en la región RBD como las descritas para las VOCs. Sin embargo, parece facilitar la entrada del virus en la célula, probablemente favoreciendo el proceso de fusión, lo cual podría explicar por qué los virus que poseían esa mutación desplazaron a aquellos sin ella $(7,10)$.

Se ha propuesto la vigilancia genómica para monitorear la introducción de VOCs del SARS-CoV-2 en cada país (5). Para ello, muchos países con capacidades robustas de secuenciación, secuencian el genoma completo de los aislados del SARS-CoV-2 a través del tiempo, para identificar las variantes y linajes circulantes. Esta secuenciación se realiza generalmente mediante plataformas de secuenciación de última generación (11). Existen también métodos rápidos, como $\mathrm{PCR}$ en tiempo real con sondas específicas para la identificación de mutaciones características de estas variantes. En el Laboratorio de Virología Molecular del Instituto Venezolano de Investigaciones Científicas (IVIC), se desarrolló un método alternativo rápido basado en el uso de enzimas de restricción de un fragmento de PCR de la región de la RBD, para la identificación de dos de esas mutaciones claves: la E484K y la L452R $(12,13)$. Otros métodos se basan en la secuenciación parcial de un pequeño fragmento del genoma, que concentra las mutaciones claves de estas variantes $(11,14)$.

En Venezuela, se ha adoptado una estrategia racional, basada en secuenciación parcial del genoma y dirigida a identificar las mutaciones claves que caracterizan las VOIs y VOCs (11). Esta estrategia permite analizar de forma rápida un mayor número de muestras e identificar potenciales variantes, cuya circulación se confirma por secuenciación del genoma completo (11). La obtención del genoma completo consiste en la generación de 16 amplicones solapados, de aproximadamente 2000 pb, que cubren todo el genoma del SARS-CoV-2. Se realiza la librería con la mezcla de esos amplicones para la secuenciación de última generación o NGS, utilizando una plataforma iSeq 100 (11). Esta estrategia nos ha permitido obtener ya un centenar de genomas completos del virus.

En 2021, las variantes que se han identificado en Venezuela hasta Octubre son:

1) 3 de las 4 VOCs:

- la Gamma, identificada desde principios del año 2021 y que rápidamente se difundió y predominó en todo el país.

- Luego se identificó la Alpha, en un caso en Marzo en Miranda y en Mayo varios casos en Apure, Barinas y uno en Lara
- y a finales de Junio, se confirmó la circulación de la VOC Delta, en Miranda y un caso aislado en Zulia. Esta variante predomina actualmente en la Región central del país.

2) las dos VOls:

- la Lambda, denominada también variante andina, que emergió en Perú, hasta ahora presente en nuestro país en casos aislados

- la Mu, variante que emergió en Colombia y que ha ido desplazando a la VOC Gamma en los estados occidentales.

El comportamiento de estas variantes es muy dinámico. La variante Delta ya predomina prácticamente en todo el mundo, con la excepción quizá de algunos países de Latinoamérica, donde todavía circulan algunas VOCs y VOls como la Gamma o la Mu (https://ourworldindata.org/ grapher/covid-variants-area?country). Sin embargo, se espera que la VOC Delta también predomine muy pronto en toda Latinoamérica.

Vacunación y variantes del SARS-CoV-2. El surgimiento de las variantes ha provocado una reducción de la efectividad de las vacunas para prevenir la infección por SARSCoV-2. Sin embargo, las vacunas siguen reduciendo la probabilidad de infección, aún con las variantes y sobre todo protegen significativamente contra la progresión grave de la enfermedad $(15,16)$.

Otro aspecto que concierne la inmunidad a las variantes tienen que ver con el concepto de inmunidad híbrida. Se ha observado que los individuos vacunados que luego se infectan desarrollan una inmunidad (denominada híbrida) de mayor intensidad y amplitud (capaz de proteger contra distintas variantes) que la obtenida solo con la vacunación o con una infección natural única (17). Estos resultados refuerzan la importancia de la vacunación para la protección contra la infección por SARS-CoV-2.

\section{CONCLUSIONES}

La circulación de variantes en los países es un proceso muy dinámico y Venezuela no escapa de esta realidad. Aunque no hay diferencias en el tratamiento dependiendo de la variante infectante, el incremento en la transmisión de unas variantes sobre otras y el posible incremento en la patogenicidad, afirma que es importante: 1) continuar la vigilancia genómica de este virus en el país, 2) mantener las medidas de prevención, aún entre las personas vacunadas y 3) acelerar la vacunación para garantizar en el menor tiempo la mayor cobertura posible de población vacunada. Todas estas medidas combinadas son las que nos permiten reducir la incidencia y eventualmente lograr controlar este grave flagelo.

Agradecimientos. Los estudios reseñados en esta revisión fueron financiados por el Ministerio del Poder Popular para Ciencia y Tecnología. 


\section{REFERENCIAS BIBLIOGRÁFICAS}

1. Pujol FH, Zambrano JL, Jaspe R, Loureiro CL, Vizzi E, Liprandi $\mathrm{F}$, et al. Biología y evolución del coronavirus causante de la COVID-19. Rev Soc Venezol Microbiol 2020; 40:63-70.

2. Phan T. Genetic diversity and evolution of SARS-CoV-2. Infect Genet Evol 2020; 81:104260. https://doi.org/10.1016/j. meegid.2020.104260.

3. O'Toole A, Scher E, Underwood A, Jackson B, Hill V, McCrone JT, Colquhoun R, Ruis C, Abu-Dahab K, Taylor B, Yeats C, Du Plessis L, Maloney D, Medd N, Attwood SW, Aanensen DM, Holmes EC, Pybus OG, Rambaut A. Assignment of epidemiological lineages in an emerging pandemic using the pangolin tool. Virus Evolution 2021. 7: en prensa. https://doi. org/10.1093/ve/veab064

4. Pujol FH, Esparza, J. COVID-19: virus, variantes y vacunas. Bol Acade Cienc Fis Matemat Natur 202; LXXXI: 1-10.

5. World Health Organization. Tracking SARS-CoV-2 variants. 2021. Disponible en: https://www.who.int/en/activities/trackingSARS-CoV-2-variants/

6. Altmann DM, Boyton RJ, Beale R. Immunity to SARS-CoV-2 variants of concern. Science 2021; 371(6534):1103-1104. https://doi.org/10.1126/science.abg7404.

7. Winger A, Caspari T.The Spike of Concern-The Novel Variants of SARS-CoV-2. Viruses. 2021; 13(6):1002. https://doi: $10.3390 / v 13061002$.

8. WHO. COVID-19 Weekly Epidemiological Update. Edition 58, published 21 September 2021.

9. Loureiro CL, Jaspe RC, D Angelo P, Zambrano JL, Rodriguez L, Alarcon V, et al. SARS-CoV-2 genetic diversity in Venezuela: Predominance of D614G variants and analysis of one outbreak. PLoS One. 2021; 16(2):e0247196. https://doi: 10.1371/journal. pone.0247196.

10. Korber B, Fischer WM, Gnanakaran S, Yoon H, Theiler J, Abfalterer W, et al. Tracking Changes in SARS-CoV-2 Spike: Evidence that D614G Increases Infectivity of the COVID-19 Virus. Cell. 2020; 182:812-827.e19. https://doi.org/10.1016/j. cell.2020.06.043 PMID: 32697968.
11. Jaspe RC, Sulbaran $Y$, Loureiro $C L, D$ Angelo $P$, Rodriguez L, Garzaro DJ, et al. Introduction and rapid dissemination of SARS-CoV-2 Gamma Variant of Concern in Venezuela. Infect Genet Evol. https://doi.org/10.1016/j.meegid.2021:105147.

12. Jaspe RC, Sulbaran $Y$, Loureiro CL, D Angelo P, Rodriguez L, Garzaro DJ, et al. Importance of mutations in amino acid 484 of the Spike protein of SARS-CoV-2: rapid detection by restriction enzyme analysis. Invest Clin 2021; 62(Suppl. 2): 18 - 26. https://doi.org/10.22209/IC.v62s2a02.

13. Jaspe RC, Sulbaran $Y$, Loureiro CL, D' Angelo P, Rodriguez L, Garzaro D, et al. A simple method for detection of mutations in amino acid 452 of the Spike protein of SARS-CoV-2 using restriction enzyme analysis. Invest. Clin. 62: https://doi. org/10.22209/IC.v62n4a07.

14. Bezerra MF, Machado LC, De Carvalho VDCV, Docena C, Brandão-Filho SP, Ayres CFJ, et al. A Sanger-based approach for scaling up screening of SARS-CoV-2 variants of interest and concern. Infect Genet Evol. 2021; 92:104910. https://doi: 10.1016/j.meegid.2021.104910.

15. Tao K, Tzou PL, Nouhin J, Gupta RK, de Oliveira T, Kosakovsky Pond SL, et al. The biological and clinical significance of emerging SARS-CoV-2 variants. Nat Rev Genet. 2021:1-17. https://doi: 10.1038/s41576-021-00408-x

16. Wahid M, Jawed A, Mandal RK, Dailah HG, Janahi EM, Dhama $\mathrm{K}$, et al. Variants of SARS-CoV-2, their effects on infection, transmission and neutralization by vaccine-induced antibodies. Eur Rev Med Pharmacol Sci. 2021; 25(18):5857-5864. https:// doi: 10.26355/eurrev_202109_26805.

17. Andreano E, Paciello I, Piccini G, Manganaro N, Pileri P, Hyseni I, et al. Hybrid immunity improves $B$ cells and antibodies against SARS-CoV-2 variants. Nature. 2021 Oct 20. https://doi: 10.1038/s41586-021-04117-7. 